\title{
FLÓRULA RUDERAL DA CIDADE DE LAVRAS, MG ${ }^{1}$
}

\author{
Manuel Losada Gavilanes ${ }^{2}$ \\ Cláudio Ney D'Angieri Filho ${ }^{2,3}$
}

Recebido em 16-9-91. Aceito em 17-2-92.

RESUMO: Efetuou-se olevantamento e a identificação das plantas ruderais ocorrentes na cidade de Lavras, MG. Foram identificadas 175 espécies, representando 122 gêneros, pertencentes a 41 famílias. As famílias. Compositae, Leguminosae, Graminese, Malvaceae, Convolvulaceae, Cyperaceae, Euphorbiaceae e Amaranthaceae, são as que apresentaram o maior número de espécies. O material foi coletado em áreas ajardinadas, beiras de caminhos, calçadas, muros e cercas, e em terrenos baldios.

Palavras-chave: Plantas ruderais; Invasoras; Lavras; Minas Gerais.

ABSTRACT - A survey and identification of ruderal plants occuring in Lavras City, state of Minas Gerais, Brazil, was carried out. One hundred and seventy five species, representing 122 genera belonging to 41 families were indentified. Families Compositae, Leguminosae, Gramineae, Malvaceae, Convolvulaceae, Cyperaceae, Euphorbiaceae, and Amaranthaceae presented the greatest numbers of species among all families. All plant materials were collected in garden areas, along roadsides and streets, walls and fences, and wastelands.

Key words: Ruderal Plants; Weeds; Minas Gerais; Brazil.

\section{Introdução}

O homem, quando da conquista de novas terras, leva consigo seus implementos agrícolas, animais e plantas domesticadas, e junto a estes, frutos e/ou sementes de plantas consideradas indesejáveis.

\footnotetext{
${ }^{1}$ Trabalho apresentado durante o XLII Congresso Nacional de Botânica, realizado em Goiânia, Goiás, em janeiro de 1991.

${ }^{2}$ Departamento de Biologia - DBI da Escola Superior de Agricultura de Lavras - ESAL. Caixa Postal, 37. 37.200 Lavras, MG.

${ }^{3}$ Bolsista de Iniciaçāo Científica do CNPq. DBI - ESAL, Lavras, MG.
} 
Quando da instalação de seus núcleos de colonização, e das culturas de um modo geral, dispersa, concomitantemente, os diásporos dessas plantas que, com o correr dos tempos, invadem não só os terrenos baldios, como também, infiltram-se na vegetação nativa. No primeiro caso, elas são denominadas de plantas ruderais e/ ou invasoras e, quando nas culturas, de plantas daninhas.

Essas plantas com sua rusticidade e grande adaptabilidade aos diversos climas e solos (Marzoca, 1957; Blanco, 1982; Brandão et al., 1982; Pitelli, 1985), além dos meios sofisticados de dispersão (Brandão \& Cunha, 1982), instalam-se em moradias definitivas, crescendo e multiplicando.

O conhecimento dos componentes dessa flora invasora é de suma importância pois, além da competição promovida às culturas, quando devem ser erradicados ou controlados, mostram aspectos positivos, podendo ser utilizados como plantas comestíveis (Brandão et al., 1985), como plantas medicinais (Brandão et al. 1985; Gavilanes et al., 1987), como plantas apícolas (Brandão et al., 1984), como plantas ornamentais (Brandão et al., 1985), como plantas forrageiras (Ferreira et al., 1984; Brandão et. al., 1985), e outros fins (Brandão et al., 1985).

Devido aos seus aspectos tanto negativos como positivos, é importante c'le essa flora seja estudada de maneira mais restrita (município ou cidade), visando-se obter, localmente, informações quanto aos seus prováveis usos.

\section{Material e métodos}

\section{A. Caracterização da Região estudada}

A cidade de Lavras está localizada na Região Sul do Estado de Minas Gerais, fazendo parte da Microrregião 199 (Alto do Rio Grande). Sua posição geográfica é determinada pelas coordenadas $21^{\circ} 14^{\prime} 30^{\prime \prime}$ de latitude Sul e $45^{\circ} 00^{\prime} 10^{\prime \prime}$ de longitude Oeste. As cotas altimétricas variam de 900 a 1200 metros. O clima é do tipo Cwb de Köppen, com pluviosidade média anual de $1.411,5 \mathrm{~mm}$; a temperatura média anual é de $19.3^{\circ} \mathrm{C}$ e a umidade relativa média de $77.7 \%$. A rede de drenagem faz parte do Rio Grande e alguns cursos de água, entre eles o Cervo e o Capivari, destacam-se por sua extensão e por determinar o limite com municípios vizinhos. A cobertura vegetal do município é constituída por duas formações distintas: a) a florestal, representada por mata de galeriạ, ou mata ciliar, constituída por prolongamentos da floresta atlânticá através do pla nalto central, e que se apresenta sob forma de capões esparsos; b) a campestre, constituída pelo cerrado, pelo campo rupestre e pelo campo limpo. Ccmo formações antrópicas há as capoeiras e capoeirões, assim como os campos antrópicos (Gavilanes \& Brandão, 1991).

\section{B. Metodologia}

Foram coletadas e identificadas as espécies, objeto do estudo, nas áreas de interesse, a saber: áreas ajardinadas, beiradas de caminhos, muros e cercas, terrenos 
baldios e loteamento em expansão, dentro do perímetro urbano da cidade de Lavras, situada na Microrregião 199 (Alto do Rio Grande) no Sul do Estado de Minas Gerais.

O material básico para este trabalho está sendo coletado desde 1986, encontrando-se devidamente catalogado e registrado no Herbarium ESAL (Herbário do Departamento de Biologia-DBI da Escoa Superior de Agricultura de Lavras-ESAL).

A identificação do material coletado vem sendo feita com o auxílio das chaves de Barroso (1946), Barroso et al. $(1978,1984)$, e de manuais como os de Bacchi et. al. (1984), Leilão Filho et. al. (1972, 1975), Lorenzi (1982) e Marzoca (1957).

Por motivos de ordem prática, as famílias, bem como os gêneros e espécies, são citadas em ordem alfabética. São também mencionados os nomes populares que as espécies recebem na região de estudo. Após a citação, é feita menção a apenas uma coleta como respectivo número de registro no Herbário, para se evitar uma lista muito extensa de citações.

\section{Resultados e Discussão}

Até o presente momento, foram identificadas, como plantas ruderais, na cidade de Lavras-MG., 175 espécies, englobadas em 122 gêneros, pertencentes a 41 famílias botânicas. As plantas em questão encontram-se listadas na Tabela 1. Na Tabela 2 são relacionadas as famílias e seus respectivos números de gêneros e de espécies.

As espécies foram enquadradas como ruderais, apenas quando coletas em no mínimo três locais distintos, dentro do perímetro urbano, conforme preconizado por Cervi et al. (1987).

São poucas as referências bibliográficas de ocorrências de plantas invasoras, ou ruderais, em áreas urbanas. Cervi \& Guimarães (1975) e Cervi et al. (1987, 1988), cuidaram daquelas da cidade de Curitiba; Lisboa (1956) estudou as de Ouro Preto; Pereira \& Agarez $(1974,1977)$, complementando o trabalho de Kuhlmann et al. (1947), fazem referências a espécies que ocorrem no Rio de Janeiro; Pott \& Pott (1985), estudaram as ruderais da Cidade de Corumbá, MS.

Os autores acima mencionam 141, 116, 23 e 273 espécies para Curitiba, Ouro Preto, Rio de Janeiro e Corumbá, respectivamente; mostram-se comuns aos trabalhos citados, as espécies: Amaranthus spinosus, Momordica charantia, Portulaca oleracea, Scoparia dulcis, Corchorus hirtus, Asclepiascurassavica, Chenopodium ambrosioides, Ageratum conyzoides, Bidens pilosa, Solidago microglossa, Leonotis nepetaefolius, Leonurus sibiricus, Chaptalia nutans, Elephantopus mollis, Galinsoga parviflora, Borreria verticillata, Verbena bonariensis, Taraxacum officinale, Elvira biflora, Oxalis oxyptera, Pyrostegia venusta, Acanthospermum australe, Galinsoga ciliata, Richardia brasiliensis, Eleusine indica, Plantago major, Alternathera ficoidea, Emilia sonchifolia, Cynodon dactylon, Lantana camara, Sida urens, Amaranthus deflexus, Solanum sisymbriifolium, Starchytarphetta cayenensis, Ipomoea purpurea, Sida rhombifolia, Boussingaultia baselloides, Rubus rosaefolius, Polygala paniculata e Ricinus communis. 
As demais têm ocorrência esporádica em um ou outro dos locais mencionados, havendo espécies específicas para as regiões estudadas, dependendo da localização e clima.

Foi constatada a presença de espécies que raramente são mencionadas, ou ainda não foram citadas, como plantas daninhas e/ou ruderais, no Estado de Minas Gerais, segundo Brandão et al. (1990, 1991). As espécies em questão são: Thumbergia fragrans, Boussingaultia baselloides, Bidens pilosa var. radiata, Gulinsoga ciliata, Hypochoeris brasiliensis, Melampodium divaricatum, Parthenim hysterophorus, Trixis antimenorhaea, Cuscutra racemosa, Cyperus iria, Mirabilis jalapa, Oxalis hirsutissima, Rubus brasiliensis, Rubus rosaefolius, Pilea microphylla e Cyperus brevifolius.

Tabela 1 - Relação das plantas ruderais que ocorrem na cidade de Lavras-MG.

\begin{tabular}{|c|c|c|}
\hline FAMILIA/NOME CIENTIFICO & NOME(S) POPULAR(ES) & $\begin{array}{c}\mathrm{N}^{2} \mathrm{DE} \\
\text { REGISTRO } \\
\text { HERBÁRIO } \\
\text { ESAL }\end{array}$ \\
\hline \multicolumn{3}{|l|}{ ACANTHACEAE } \\
\hline Thumbergia alata Bojer & BUNDA-DE-MULATA & 07914 \\
\hline Thumbergia fragrans Roxb. & MARIA-SEM-VERGONHA-BRANCA & 07208 \\
\hline \multicolumn{3}{|l|}{ AMARANTHACEAE } \\
\hline Alternanthera brasiliana $(\mathrm{L}$.$) O. Kuntze$ & CARRAPICHINHO & 07929 \\
\hline Alternanthera ficoidea (L.) R. Br. & APAGA-FOGO; CARRAPICHO & 06630 \\
\hline Amaranthus deflexus $\mathrm{L}$ & CARURU & 08821 \\
\hline Amaranthus hybridus $\mathrm{L}$. & CARURU-VERMELHO & 06574 \\
\hline Amaranthus lividus $\mathrm{L}$. & CARURU-RASTEIRO & 07899 \\
\hline Amaranthus retroflexus $\mathrm{L}$. & CARURU & 07309 \\
\hline Amaranthus spinosus $\mathrm{L}$. & CARURU-DE-ESPINHO & 06562 \\
\hline \multicolumn{3}{|l|}{ ASCLEPIADACEAE } \\
\hline Asclepias curassavica $\mathrm{L}$. & OFICIAL-DE-SALA; PAININHA & 09133 \\
\hline \multicolumn{3}{|l|}{ BASELLACEAE } \\
\hline Boussingaultia baselloides H.B.K. & BERTALHA & 09180 \\
\hline \multicolumn{3}{|l|}{ BIGNONIACEAE. } \\
\hline Pyrostegia venusta (Ker-Gawl.) Miers. & CIPÓ-DE-SĀO JOĀO & 07948 \\
\hline \multicolumn{3}{|l|}{ BORAGINACEAE } \\
\hline Heliotropium indicum L. & CRISTA-DE-GALO & 06945 \\
\hline \multicolumn{3}{|l|}{ CHENOPODIACEAE } \\
\hline Chenopodium ambrosioides $\mathrm{L}$. & ERVA-DE-SANTA MARIA & 05830 \\
\hline \multicolumn{3}{|l|}{ COMMELINACEAE } \\
\hline Commelina nudiflora $\mathrm{L}$ & TRAPOERABA; CAPIM GOMOSO & 06641 \\
\hline Tradescantia elongata Meyer & TRAPOERABA & 09204 \\
\hline
\end{tabular}


Cont. Tabela 1

№ $\mathrm{DE}$

FAMILIA/NOME CIENTÍFICO

NOME(S) POPULAR(ES)

REGISTRO

HERBÁRIO

ESAL

COMPOSITAE

Acanthospermum australe (Loef.) O. Kuntze

CARRAPICHO-DE-CARNEIRO 06662

Achyrocline satureoides (Lam.) DC.

MACELA

08598

Ageratum conyzoides L.

Ambrosia polystachya DC.

Baccharis punctulata DC.

Bidens pilosa $\mathrm{L}$.

Bidens pilosa L. var. radiata Schult. - Bip.

Blainvillea biaristata DC.

Blainvilleu rhomboideu Cass.

Centratherum punctatum Cass.

Chaptalia nutans (L.) Polak

MENTRASTO

07762

CRAVO-DA-ROÇA; CRAVORANA 07931

CARQUEJA

06588

PICÃO; PICÃO-PRETO 07834

PICĀO 07531

PICĀO-GRANDE

ERVA-PALHA

cos:s =

06632

PERPETUA-DO-MATO 09138

Conyza bonariensis (L.) Cronq.

LINGUA-DE-VACA

07913

BUVA; MARGARIDINHA-DO-CAMPO

07532

Cosmus caudatus H.B.K.

AMOR-DE-MOÇA

09337

AMOR-DE-MOÇA

09136

Cosmus sulphureus Cav.

Elephantopus mollis H.B.K.

ERVA-GROSSA

07530

MARGARIDA-VERDE 09334

Elvira biflora (L.) DC.

Emilia sonchifolia DC.

Erechtites valerianaefolia DC.

PINCEL; ERVA-PINCEL

03817

CAPIÇOBA 07240

Gulinsoga ciliata (Raf.) Blake

FAZENDEIRO

06552

PICĀO-BRANCO 07835

Galinsoga parviflora Cav.

Gamochaeta pensylvanica (Willd.) Cabr.

MACELA; MACIO; MELOSO 07314

ALMEIRĀO-DO-CAFEZAL 08964

ESTRELINHA 08295

LÍNGUA-DE-VAGA 08711

LOSNA-BRANCA; COENTRO-BRAVO 10125

VERBASCO 07985

BOTÃO-DE-OURO 07571

ERVA-LANCETA 08421

SERRALHA 07317

CRAVO-DE-DEFUNTO 07233

RABO-DE-ROJÃO 07529

DENTE-DE-LEÃO 07316

MARGARIDÃO 10027

ERVA-ANDORINHA; SOLIDÔNIA 11991

ASSA-PEIXE $\quad 08365$

ASSA-PEIXE 07415

ESPINHO-DE-CARNEIRO 07573

ZINIA $\quad 08074$

\section{CONVOLVULACEAE}

Ipomoea cairica (L.) Sweet

Ipomoea coccinea $\mathrm{L}$.

Ipomoea cynanchifolia Meissn.

Ipomoea hederifolia $\mathrm{L}$.

Ipomoea longicuspis Meissn.

CAMPAINHA; CORDA-DE-VIOLA 
Cont. Tabela 1

Cont. Tabela 1

FAMILIA/NOME CIENTÍFICO

NOME(S) POPULAR(ES)

$\mathrm{N}^{\circ} \mathrm{DE}$

REGISTRO

HERBÁRIO

ESAL

Ipomoea purpurea Lam.

CAMPAINHA; JETIRANA-ROXA

06554

Ipomoea quamoclit $\mathrm{L}$.

CIPO-ESQUELETO; ESQUELETO

02291

Merremia macrocalyx (Ruiz \& Pav.) O'Donnel

CAMPAINHA-BRANCA; JETIRANA

06557

CRUCIFERAE

Lepidium pseudodidymum Thell.

MASTRUZ

08819

Lepidium ruderale $\mathrm{L}$.

Lepidium virginicum $\mathrm{L}$.

Raphanus raphanistrum $\mathrm{L}$.

MASTRUÇO

07932

MENTRASTO

06582

NABIÇA

09125

Sinapsis arvensis $\mathrm{L}$.

MOSTARDA

08292

\section{CUCURBITACEAE}

Momordica charantia $\mathrm{L}$.

MELĀO-DE-SÃO CAETANO

08856

\section{CUSCUTACEAE}

Cuscuta racemosa Mart.

CIPÓ-СHUMBO

06556

\section{CYPERACEAE}

Cyperus brevifolius (Rottb.) Hassk.

Cyperus iria L.

Cyperus rotundus $\mathrm{L}$.

Dichromena ciliata Vahl.

Eleocharis elegans (H.B.K.) Røm. et Schult.

Fimbristylis diphylla (Retz.) Vahl.

Fimbristylis miliacea (L.) Vahl.

TIRIRICA

11988

TIRIRICA

11990

TIRIRICA

11992

CAPIM-ESTRELA

06329

JUNCO-MANSO

10821

FALSO-ALECRIM-DA-PRAIA

11989

FALSO-COMINHO

11993

\section{EUPHORBIACEAE}

Croton glandulosus (L.) Muell. Arg.

Croton lundianus (F. Diedr.) Muell. Arg.

GERVĀO-BRANCO

07322

Euphorbia brasiliensis Lam.

Euphorbia heterophylla L.

Euphorbia prostata Ait.

Phyllanthus corcovadensis Muell. Arg.

Ricinus communis $\mathrm{L}$.

GERVÃO-BRANCO

07933

LEITEIRA

11986

AMENDOIM-BRAVO; LEITEIRA

06639

QUEBRA-PEDRA-RASTEIRO

12008

ERVA-POMBINHA; QUEBRA-PEDRA

08817

MAMONA; CARRAPATEIRA

06660

\section{GRAMINEAE}

Brachiaria plantaginea (Link.) Hitch.

Coix lacrima-jobi L

Cymbopogon densiflorus

Cynodon dactylon (L.) Pers.

Digitaria insularis (L.) Mez

Eleusine indica (L.) Gaertn.

Eragrostis acuminata Doell

Melinis minutiflora Beauv.

Phalaris canariensis $\mathrm{L}$.

Rhynchelitrum repens (Willd.) Hubbard

CAPIM-MARMELADA; MARMELADA

GRAMA-SEDA; CAPIM-FINO 07947

CAPIM-AMARGOSO 09178

CAPIM-DE-PÉ-GALINHA 01952

CAPIM-MIMOSO; CAPIM-FINO 00571

CAPIM-GORDURA; CAPIM-MELOSO $\quad 06560$

ALPISTE 07928

CAPIM-FAVORITO 06571 
Flórula ruderal...

Cont. Tabela 1

$\begin{array}{lc}\text { FAMILIA/NOME CIENTIFICO } & \text { REME(S) POPULAR(ES) } \\ \text { HERBÁRIO } \\ \text { ESAL }\end{array}$

Setaria geniculata (Lam.) Beauv.

CAPIM-RABO-DE-RATO

07543

Sorghum halepense (L.) Pers.

CAPIM-MASSAMBARÁ

05032

\section{LABIATAE}

Hyptis reticulata Mart. ex Benth.

HORTELÃ

08850

Hyptis suaveolens Poit.

Leonotis nepetaefolius (L.) R. Br.

Leonurus sibiricus $\mathrm{L}$.

Marsypianthes chamaedrys (Vahl.) Kuntze

Salvia splendens Sellow

MENTRASTO

07949

CORDÃO-DE-FRADE

12011

CORDÃO-DE-SÃO FRANCISCO

06572

HORTELĀ-DO-BRASIL

07324

CARDEAL-DO-BRASIL

12017

\section{LEGUMINOSAE}

Aeshynomene elegans Schlt. et Cham.

CARRAPICHINHO

06581

Aeshynomene falcata (Poir.) DC.

CARRAPICHINHO

09188

Aeschynomene paniculata $\mathrm{L}$

CARRAPICHINHO

09189

Chamaecrista nictitans L. subsp. patellaria

(Collad) Irwin \& Barneby

PENINHA 08323

Chamaecrista rotundifolia (Pers.) Greene

Clitoria glycinioides DC.

Crotalaria anagyroides H.B.K.

Crotalaria lanceolata $\mathrm{E}$. Mey

Desmodium barbatum (L.) Benth.

Desmodium canum (Gmel) Schinz et Thell.

Desmodium purpureum (Mill.) Fawc. et Rendle

ERVA-CORAÇÃO

08327

MATA-CABRA

09193

CHOCALHO; CASCAVEL $\quad 07422$

CHOCALHO; CASCAVEL 08328

BARBADINHO 08325

CARRAPICHO 08324

CARRAPICHO 08361

Mimosa invisa Mart.

Mimosa pudica L

ARRANHA-CANELA

04376

SENSITIVA; MUCHA-CADELA

08290

FEDEGOSO; MATA-PASTO

06657

ALFAFA-DO-CAMPO

08366

Stylosanthes guyanensis (Aubl.) DC.

Stylosanthes leiocarpa Vog.

Stylosanthes viscosa $\mathrm{Sw}$.

Zornia latifolia $\mathrm{Sm}$.

ALFAFA-DO-CAMPO

03925

ALFAFA-DO-CAMPO

07945

URINÁRIA

09187

\section{LILIACEAE}

Nothoscordum fragrans Kunth.

ALHO-BRAVO

06566

\section{LOGANIACEAE}

Buddleya brasiliensis Jacq.

BARBASCO

07425

\section{LYTHRACEAE}

Cuphea cartagenensis (Jacq.) MacBride

Cyphea mesostemon Koehne

SETE-SANGRIAS; MATA-PASTO

11998

SETE-SANGRIAS; MATA-PASTO

09196

06193

\section{MALVACEAE}

Hibiscus sabdariffa L.

Malvastrum coromandelianum (L.) Garcke

AZEDIN 
Cont. Tabela 1

\section{FAMILIA/NOME CIENTIFICO}

NOME(S) POPULAR(ES)

$\mathrm{N}^{2} \mathrm{DE}$

REGISTRO

HERBÁRIO

ESAL

Pavonia sagitatta St. Hil

MALVAVISCO

07908

Sida carpinifolia L.f.

GUANXUMA

10516

Sida cordifolia L.

VASSOURA; GUANXUMA

07915

Sida linifolia Cav.

MALVA-FINA

06672

Sida rhombifolia L.

GUANXUMA; VASSOURA

12003

Sida urens $\mathrm{L}$.

MALVA

07757

Urena lobata $\mathrm{L}$.

GUANXUMA-ROXA

07957

NYCTAGINACEAE

Mirabilis jalapa $\mathrm{L}$.

MARAVILHA

08333

ONAGRACEAE

Ludwigia suffruticosa (L.) Ha،a

CRUZ-DE-MALA

07113

OXALIDACEAE

Oxulis hirsutissima Mart. et Zucc.

TREVO-PELUDO

08293

Oxalis oxyptera Prog.

AZEDINHA; TREVO

09171

PLANTAGINACEAE

Plantago major L.

Plantago tomentosa Lam.

TANCHAGEM

08326

TANCHAGEM

10698

POLYGALACEAE

Polygala paniculata L.

BARBA-DE-SÃO PEDRO

07100

Polygala violacea Aubl.

VIOLETA; ROXINHA

08321

\section{POLYGONACEAE}

Polygonum acre H.B.K.

Polygonum capitatum H.B.K.

ERVA-DE-BICHO

07926

CABEÇA-ROSA

11999

Polygonum hidropiperoides Mich.

ERVA-PULGUEIRA

12000

\section{PORTULACACEAE}

Portulaca oleracea L

Talinum patens (Jacq.) Willd.

BELDROEGA

12012

LINGUA-DE-VAGA

12004

\section{ROSACEAE}

Rubus brasiliensis Mart.

Rubus rosaefolius Smith.

AMORA-SILVESTRE $\quad 05971$

AMORA-SILVESTRE $\quad 08140$

\section{RUBIACEAE}

Borreria latifolia (Aubl.) Schum.

Borreria suaveolens G.F.W. Meyer

Borreria verticillata (L) G.F.W. Meyer

Manettia ignita Schum.

Relbunium hirsutum Schum.

Richardia brasiliensis Gomez

POAIA; QUEBRA-TIJELA

POAIA-DO-CERRADO

CABEÇA-BRANCA

FLOR-DE-SANTO ANTONIO

POAIA-BRANCA 
Flórula ruderal...

Cont. Tabela 1

$\mathrm{N}^{2} \mathrm{DE}$

FAMILIA/NOME CIENTIFICO

NOME(S) POPULAR(ES)

REGISTRO

HERBÁRIO

ESAL

SAPINDACEAE

Serjania caracasana (Jacq.) Willd.

TIMBO

07942

SCROPHULARIACEAE

Scoparia dulcis L.

VASSOURINHA

07714

SOLANACEAE

Datura suaveolens H. et. B. ex Willd.

TROMBETEIRA

07916

Datura stramonium L.

FIGUEIRA-DO-INFERNO12015

Physalis angulata L.

JOÃ-DE-CAPOTE

06667

Solanum aculeatissimum Jacq.

JOÃ; JOĀ-BRAVO

06644

Solanum americanum Mill.

MARIA-PRETINHA

12002

Solanum sisymbrïfolium Lam.

JOĀ; JOĀ-DE-ROÇA

06643

\section{STERCULIACEAE}

Waltheria indica $\mathrm{L}$.

MALVA-PELUDA

06569

TILIACEAE

Corchorus hirtus L.

VASSOURA

07112

Triumfetta semitriloba Jacq.

CARRAPICHINHO-MIUDO

07954

TROPAEOLACEAE

Tropaeolum majus $\mathrm{L}$.

CAPUCHINHA; CHAGAS

08853

\section{TURNERACEAE}

Turnera ulmifolia $\mathrm{L}$.

CHANANA

07357

\section{UMBELLIFERAE}

Apium leptophyllum (Pers.) F. Muell.

MASTRUÇO

11985

Hydrocotile umbellata $\mathrm{L}$.

ERVA-CAPITĀO

11997

Foeniculum vulgare Mill.

ERVA-DOCE

05319

URTICACEAE

Pilea microphylla (L.) Liebm.

BRILHANTINA

11996

\section{VERBENACEAE}

Lantana camara L.

CAMBARĀ; ERVA-CHUMBINHO

07959

Lantana lilacina Desf.

MILHO-DE-GRILO

07209

Starchytarphetta cayenensis (L.C. Rich.) Vahl.

GERVÃO; GERVÃO-AZUL

09120

Verbena bonariensis L

CAMBARÃ-DE-CAPOEIRA

09132 
Tabela 2 - Famílias, números de gêneros e de espécies de plantas ruderais ocorrentes na cidade de Lavras, MG.

\begin{tabular}{|c|c|c|}
\hline FAMÍLIA & GÊNERO & ESPÉCIE \\
\hline ACANTHACEAE & 1 & 2 \\
\hline AMARANTHACEAE & 2 & 7 \\
\hline ASCLEPIDACEAE & 1 & 1 \\
\hline BASELLACEAE & 1 & 1 \\
\hline BIGNONIACEAE & 1 & 1 \\
\hline BORAGINACEAE & 1 & 1 \\
\hline CHENOPODIACEAE & 1 & 1 \\
\hline COMMELINACEAE & 2 & 2 \\
\hline COMPOSITAE & 33 & 38 \\
\hline CONVOLVULACEAE & 2 & 8 \\
\hline CRUCIFERAE & 3 & 5 \\
\hline CUCURBITACEAE & 1 & 1 \\
\hline CUSCUTACEAE & 1 & 1 \\
\hline CYPERACEAE & 4 & 7 \\
\hline EUPHORBLACEAE & 4 & 7 \\
\hline GRAMINEAE & 12 & 12 \\
\hline LABIATAE & 5 & 6 \\
\hline LEGUMINOSAE & 8 & 18 \\
\hline LILIACEAE & 1 & 1 \\
\hline LOGANIACEAE & 1 & 1 \\
\hline LYTHRACEAE & 2 & 3 \\
\hline MALVACEAE & 5 & 9 \\
\hline NYCTAGINACEAE & 1 & 1 \\
\hline ONAGRACEAE & 1 & 1 \\
\hline OXALIDACEAE & 1 & 2 \\
\hline PLANTAGINACEAE & 1 & 1 \\
\hline POLYGALACEAE & 1 & 2 \\
\hline POLYGONACEAE & 1 & 3 \\
\hline PORTULACACEAE & 2 & 2 \\
\hline ROSACEAE & 1 & 2 \\
\hline RUBIACEAE & 4 & 6 \\
\hline SAPINDACEAE & 1 & 1 \\
\hline SCROPHULARIACEAE & 1 & 1 \\
\hline SOLANACEAE & 3 & 6 \\
\hline STERCULIACEAE & 1 & 1 \\
\hline TILIACEAE & 2 & 2 \\
\hline TROPAEOLACEAE & 1 & 1 \\
\hline TURNERACEAE & 1 & 1 \\
\hline UMBELLIFERAE & 3 & 3 \\
\hline URTICACEAE & 1 & 1 \\
\hline VERBENACEAE & 3. & 4 \\
\hline TOTAL: 41 famílias & 122 gêneros & 175 espécies \\
\hline
\end{tabular}


Pretende-se, em uma próxima etapa, caracterizar as prováveis potencialidades econômicas das espécies identificadas, além de fornecer dados sobre sua morfologia, fenologia, etc.

\section{Referências Bibliográficas}

BACCHI, O.; H.F. LEITÃO FILHO \& C. ARANHA. 1984. Plantas invasoras de culturas. Campinas, Instituto Campineiro de Ensino Agrícola, v. 3. 308 p.

BARROSO, G.M.; E.F. GUIMARÃES; C.L.F. ICHASO; C.G. COSTA \& A.L. PEIXOTO. 1978. Sistemática das Angiospermas do Brasil. São Paulo, LTC/ EDUSP, v.1. 255 p.

BARROSO, G.M.; A.L. PEIXOTO, C.G. COSTA; C.L. ICHASO, E.F. GUIMARÃES \& H.C. LIMA. 1984. Sistemática das Angiospermas do Brasil. Viçosa, Imprensa Universidade da U.F.V., v. 2. 377 p.

BARROSO, L.J.1946. Chaves para a determinação de gêneros indígenas e exóticos das dicotiledôneas do Brasil. Rio de Janeiro, Min. da Agricultura/Serviço de Documentação. 2 ed. 272 p.

BLANCO, H.G. 1982. Ecologia das plantas daninhas; competição de plan-tas daninhas em culturas brasileiras. In: MARCONDES, D.A. et al. Controle integrado de plantas daninhas. São Paulo, CREA. p. 43-75.

BRANDÄO, M. \& L.H.S. CUNHA. 1982. Meios de dispersão empregados por plantas daninhas. Informe Agropecuário, Belo Horizonte, 8(87): 4-10.

BRANDÃO, M.; M.L. GAVILANES \& J.P. LACA-BUENDIA. 1990. Plantas daninhas raramente mencionadas ou não citadas como ocorrentes em Minas Gerais. Daphne, Belo Horizonte, 1(1): 22-25.

BRANDÃO, M.; J.P. LACA-BUENDIA \& M.L. GAVILANES. 1982. Principais plantas daninhas no Estado de Minas Gerais. Informe Agropecuário, Belo Horizonte, 8(87): 18-26.

BRANDÃO, M.; J.P. LACA-BUENDIA \& M.L. GAVILANES. 1991. Plantas daninhas raramente mencionadas ou não citadas como ocorrentes em Minas Gerais. Daphne, Belo Horizonte, 1(3): 13-16.

BRANDÃO, M.; M.L. GAVILANES, L.H.S. CUNHA, J.P. LACA-BUENDIA \& C. CARDOSO. 1984. Plantas consideradas daninhas para culturas como fonte de néctar e pólen. Planta Daninha, Campinas, 7(2): 1-22.

BRANDÃO, M.; M.L. GAVILANES, L.H.S CUNHA, M.A. ZURLO, \& C. CARDOSO. 1985. Novos enfoques para plantas consideradas daninhas. Informe Agropecuário, Belo Horizonte, 11(129): 3-12.

CERVI, A.C. \& O.A. GUIMARÃES. 1975. Catálogo das plantas ruderais da cidade de Curitiba. Tribuna Farmacêutica, Curitiba, 43(1-2): 1-20.

CERVI, A.C.; O.A. GUIMARÃES, R.R.B. NEGRELLE, D. SBALCHIERO \& M.C.A. CONCEIÇÃO, 1987. Catálogo das plantas ruderais da cidade de 
Curitiba-PR. Estudos preliminares - II. Revista do Setor de Ciências Agrárias, Curitiba, 9: 157-163.

CERVI, A.G.; O.A. GUIMARÃES, L.A. ACRA, R.R.B. NEGRELLI \& D. SBALCHIERO. 1988. Catálogo das plantas ruderais da cidade de Curitiba, Brasil. Estudos preliminares - III. Acta Biológica Paranaense, Curitiba, 17(1, 2, 3, 4): 109-139.

FERREIRA, M.B.; G.A.R. MACEDO \& J.P. LACA-BUENDIA. 1984. Plantas daninhas com possibilidades de forrageiras para bovinos em condições de cerrado. Planta Daninha, Campinas, 7(1): 41-48.

GAVILANES, M.L.; M. BRANDÃO \& C. CARDOSO. 1987. Levantamento das plantas daninhas utilizadas como medicinais, de uso popular. Oreádes, Belo Horizonte, 8(14/15): 34-47. (Anais do Simpósio de Plantas Medicinais do Brasil, 7, Belo Horizonte, 1982).

GAVILANES, M.L. \& M. BRANDÂO. 1991. Informações preliminares acerca da cobertura vegetal do município de Lavras, MG. Daphne, Belo Horizonte, 1(2): 44-50.

KUHLMANN, J.G.; P.OCCHIONI \& J.I.A.FALCÃO.1947. Contribuição ao estudo das plantas ruderais do Brasil. Arquivos do Jardim Botânico do Rio de Janeiro, Rio de Janeiro, 7: 43-133.

LEITÃO FILHO, H.F.; C. ARANHA \& O. BACCHI. 1972. Plantas invasoras de culturas no Estado de São Paulo. São Paulo, Hucitec. v. 1. 291 p.

LEITÃO FILHO, H.F.; C. ARANHA; O. BACCHI. 1975. Plantas invasoras de culturas no Estado de São Paulo. São Paulo, Hucitec - Agiplan. v. 2.305 p.

LISBOA, M.A. 1956. Aspectos da flora ruderal de Ouro Preto. In: Anais do V. Congresso Nacional de Sociedade Botânica do Brasil. Porto Alegre, 1954. Sociedade Botânica do Brasil, p. 133-146.

LORENZI, H. DE. 1982. Plantas daninhas do Brasil: terrestres, aquáticas, parasitas, tóxicas e medicinais. Nova Odessa, Ed. do autor. 425 p.

MARZOCA, A. 1957. Manual de malezas. Buenos Aires, INTA, 530 p.

PEREIRA, C. \& F.V. AGAREZ. 1974. Estudo das plantas ruderais do Estado da Guanabara. Leandra, Rio de Janeiro, 3/4(4-5): 67-63.

PEREIRA, C. \& F.V. AGAREZ. 1977. Estudo das plantas ruderais do Estado do Rio de Janeiro - II. Leandra, Rio de Janeiro, 6/7(7): 77-93.

PITELLI, R.A. 1985. Interferência de plantas daninhas em culturas agrícolas. Informe Agropecuário, Belo Horizonte, 11(129): 16-27.

POTT, V.J. \& A. POTT. 1985. Flórula ruderal da Cidade de Corumbá, MS. In: Anais do XXXVI Congresso Nacional de Botânica, 36, Curitiba, 1985. IBAMA, v. 2, p. 519-535. 